\title{
On the Performance of Single-Threshold Detectors for Binary Communications in the Presence of Gaussian Mixture Noise
}

\author{
Suat Bayram, Student Member, IEEE, and Sinan Gezici, Member, IEEE
}

\begin{abstract}
In this paper, probability of error performance of single-threshold detectors is studied for binary communications systems in the presence of Gaussian mixture noise. First, sufficient conditions are proposed to specify when the sign detector is (not) an optimal detector among all the single-threshold detectors. Then, a monotonicity property of the error probability is derived for the optimal single-threshold detector. In addition, a theoretical limit is obtained on the maximum ratio between the average probabilities of error for the sign detector and the optimal single-threshold detector. Finally, numerical examples are presented to investigate the theoretical results.
\end{abstract}

Index Terms-Probability of error, single-threshold detector, sign detector, Gaussian mixture noise.

\section{INTRODUCTION}

$\mathbf{I}$ $\mathrm{N}$ binary communications systems that operate over additive white Gaussian noise (AWGN) channels and under average power constraints, the average probability of error is minimized when antipodal signaling is employed. In that case, the receiver estimates the transmitted bits based on the signs of correlator (or, matched filter) outputs for equiprobable information symbols [1], [2]. In other words, the sign detector is optimal in that scenario. However, in some cases, the noise has non-Gaussian statistics due to impulsive noise or co-channel interference (CCI) [3], and it is more accurately modeled as symmetric Gaussian mixture noise [4]. For impulsive noise, the Gaussian mixture is commonly the mixture of zero-mean Gaussian processes with different variances, whereas, for CCI, it is the mixture of non-zero-mean processes with the same variance [3].

In order to improve the performance of communication systems in the presence of non-Gaussian noise, detectors can be designed based on the $L_{p}$-norm, Huber's $M$-metric, and hard/soft limiters [5]-[8]. The main idea behind those approaches is to provide robust detection algorithms that can perform well under various noise distributions. In [5], an adaptive $L_{p}$-norm metric is proposed for robust diversity combining under non-Gaussian noise and interference. In addition, an application of the adaptive $L_{p}$-norm metric to orthogonal frequency division multiplexing (OFDM) is discussed in [9]. Also, [10] studies minimax detection of a weak signal and shows that for small and large noise variances, the minimax detector reduces to $L_{2}$-norm and $L_{1}$-norm distance rules, respectively.

In the presence of Gaussian mixture noise, with zero or non-zero mean, the optimal detector that minimizes the

Paper approved by A. Zanella, the Editor for Wireless Systems of the IEEE Communications Society. Manuscript received July 27, 2009; revised January 19, 2010 and May 7, 2010.

The authors are with the Department of Electrical and Electronics Engineering, Bilkent University, Bilkent, Ankara 06800, Turkey (e-mail: \{sbayram, gezici\}@ee.bilkent.edu.tr).

Digital Object Identifier 10.1109/TCOMM.2010.091710.090124 average probability of error can be different from the sign detector in general and it can result in a decision rule with multiple threshold levels. Specifically, the optimal detector needs to calculate the likelihood ratio for each bit and compare it to a threshold [1], which can require significantly more computations than a sign detector. For receivers with limited battery life and/or computational capabilities, such as those in wireless sensor networks [11], the optimal detector may not be implemented. Therefore, the focus of this study is on the sign detector and the other single-threshold receivers, which can have significantly lower computational complexity than the optimal detector.

Although the sign detector provides a very simple receiver structure, its performance can be unacceptable for certain Gaussian mixture noise components, since it can result in high error floors (cf. Fig. 2). Therefore, the performance of the sign detector needs to be improved in some scenarios without increasing the computational complexity significantly. Hence, in this study, the aim is to optimize the detector performance under a constraint on the computational complexity. More specifically, we consider single-threshold detectors for low computational complexity and try to investigate the performance of optimal single-threshold detectors that minimize the average probability of error for a binary communications system under symmetric Gaussian mixture noise.

Although the sign detector and other single-threshold detectors have been studied extensively in the literature [1], [12], [13], no comparative study has been performed between the sign detector and the other single-threshold detectors in the presence of Gaussian mixture noise. The main contributions of this paper can be summarized as follows. First, sufficient conditions are obtained to specify when the sign detector is the optimal detector among all the single-threshold detectors. Then, it is proven that the average probability of error for the optimal single-threshold detector is a monotone increasing function of the variance of the Gaussian components in the mixture noise. It is also shown via numerical examples that the sign detector does not have this property in general. In addition, a theoretical performance comparison is made between the sign detector and the optimal single-threshold detector for small variances of the Gaussian components in the mixture noise, and it is shown that the maximum ratio between the average probabilities of error for the sign detector and the optimal single-threshold detector is equal to two. As a byproduct of this result, sufficient conditions are obtained to specify when the performance of the sign detector can or cannot be improved by replacing it with the optimal singlethreshold detector.

The remainder of the paper is organized as follows. In Section II, the signal model is introduced and the problem formulation is presented. In Section III, conditions on desired 
signal amplitude and/or the parameters of Gaussian mixture noise are derived in order to specify when the sign detector is (not) an optimal single-threshold detector. After that, the probability of error performance of the optimal singlethreshold detector is investigated, and a monotonicity property of the probability of error and the maximum improvement with respect to the sign detector are derived in Section IV. Finally, numerical examples are studied in Section V, and concluding remarks are presented in Section VI.

\section{Signal Model and Problem Formulation}

Consider a binary communications system with antipodal signaling, in which the post-filtered signal at the receiver is represented by [2]

$$
x=A b+n,
$$

where $b \in\{-1,+1\}$ represents the equiprobable binary symbol to be detected, $A>0$ is the known amplitude coefficient, ${ }^{1}$ and $n$ is the noise component, which is modeled as symmetric Gaussian mixture noise. The probability density function (PDF) of noise $n$ is given by

$$
p_{N}(x)=\sum_{i=1}^{M} \frac{w_{i}}{\sqrt{2 \pi} \sigma_{i}} \exp \left(\frac{-\left(x-x_{i}\right)^{2}}{2 \sigma_{i}^{2}}\right),
$$

where $M$ is an even number ${ }^{2}$ representing the number of Gaussian components in the mixture noise, $w_{i} \geq 0$ for $i=1, \ldots, M$, and $\sum_{i=1}^{M} w_{i}=1$. Due to the symmetry assumption, $x_{i}=-x_{M-i+1}, w_{i}=w_{M-i+1}$ and $\sigma_{i}=\sigma_{M-i+1}$ for $i=1, \ldots, M / 2$. It is assumed that the parameters of the mixture noise in (2) are known. ${ }^{3}$

The symmetric Gaussian mixture model specified above is observed in many practical scenarios [3], [14]-[16]. One important scenario is multiuser wireless communications, in which the desired signal is corrupted by interference from other users as well as zero-mean Gaussian background noise [17].

The problem is to determine the transmitted bit $b$ in (1), which can be stated as the following binary hypotheses test:

$$
\mathcal{H}_{0}: X \sim p_{N}(x+A), \quad \mathcal{H}_{1}: X \sim p_{N}(x-A),
$$

where hypotheses $\mathcal{H}_{0}$ and $\mathcal{H}_{1}$ correspond to $b=-1$ and $b=+1$ cases, respectively.

As motivated in the introduction, the aim is to investigate the performance of single-threshold detectors. A singlethreshold detector with threshold $\tau$ can be expressed as

$$
\phi_{\tau}(x)=\left\{\begin{array}{ll}
0, & x<\tau \\
1, & x>\tau
\end{array} .\right.
$$

In the case of $x=\tau$, the detector decides $\mathcal{H}_{0}$ or $\mathcal{H}_{1}$ with equal probabilities. It is well-known that the detector in (4) is not optimal in general in the presence of Gaussian mixture noise [12]. However, its main advantage is that it has very low computational complexity, which makes it very practical

\footnotetext{
${ }^{1}$ The results in the paper can be extended to $A<0$ cases as well, by switching the decision regions of the detector in (4).

${ }^{2}$ Assuming an even $M$ does not reduce the generality of the results due to the symmetry of the Gaussian mixture noise.

${ }^{3}$ In practice, a set of previous measurements can be used to estimate/learn the noise parameters.
}

for low cost applications, such as for wireless sensor networks [11].

The average probability of error for the detector in (4) can be obtained from (3) as

$$
\mathrm{P}(\tau)=\frac{1}{2} \int_{\tau}^{\infty} p_{N}(x+A) d x+\frac{1}{2} \int_{-\infty}^{\tau} p_{N}(x-A) d x .
$$

From (2), the expression in (5) becomes

$\mathrm{P}(\tau)=\frac{1}{2} \sum_{i=1}^{M} w_{i}\left[Q\left(\frac{\tau-x_{i}+A}{\sigma_{i}}\right)+Q\left(\frac{-\tau+x_{i}+A}{\sigma_{i}}\right)\right]$,

where $Q(x)=\frac{1}{\sqrt{2 \pi}} \int_{x}^{\infty} \mathrm{e}^{-t^{2} / 2} d t$ represents the $Q$-function. Since the sign detector corresponds to $\tau=0, \mathrm{P}(0)$ represents the average probability of error for the sign detector. On the other hand, the threshold value corresponding to the optimal single-threshold detector can be obtained from (6) as

$$
\begin{aligned}
\tau_{\mathrm{opt}}=\arg \min _{\tau} \sum_{i=1}^{M} w_{i}\left[Q\left(\frac{\tau-x_{i}+A}{\sigma_{i}}\right)\right. \\
\left.+Q\left(\frac{-\tau+x_{i}+A}{\sigma_{i}}\right)\right] .
\end{aligned}
$$

The average probability of error for threshold $\tau_{\text {opt }}, \mathrm{P}\left(\tau_{\text {opt }}\right)$, represents the minimum average probability of that can be achieved by a single-threshold detector.

Note that the optimization in (7) can be performed over $\tau \geq 0$ only, since it can be shown that the objective function is an even function of $\tau$ for the symmetric Gaussian mixture noise model. The optimal value in (7) can be obtained via an exhaustive $\operatorname{search}^{4}$, or via an approximate solution as in [18] for small values of $A$. The approximate solution for small $A$ can be obtained from (5) by using $p_{N}(x \pm A) \approx$ $p_{N}(x) \pm A p_{N}^{\prime}(x)$, which results in $\mathrm{P}(\tau) \approx 0.5-A p_{N}(\tau)$ after some manipulation. Then, the optimal threshold can be approximated by $\tilde{\tau}_{\mathrm{opt}}=\arg \max p_{N}(\tau)$. It should be noted that this approximate solution can be estimated from measurements (data) as the most probable value of noise, in the absence of any information about the noise PDF [18].

Remark 1: Since the optimal single-threshold detector needs to obtain the optimal threshold in (7), it has higher computational complexity than the sign detector in general. However, the optimal threshold needs to be re-calculated only when the noise statistics change (e.g., when the interference statistics change). Therefore, the optimal single-threshold detector can still have significantly lower computational complexity than the optimal detector based on likelihood ratio calculations.

\section{On the Optimality of The Sign Detector}

In this section, sufficient conditions are derived in order to determine whether or not the sign detector is optimal, among all single-threshold detectors, for the binary detection problem under symmetric Gaussian mixture noise. Those sufficient conditions carry practical importance, since they can specify when it is necessary to solve the optimization problem in (7).

\footnotetext{
${ }^{4}$ In practice, the search can be performed over an interval of $[0, A+$ $\max \left\{x_{i}\right\}$ ], where $x_{i}$ is the mean value for the $i$ th Gaussian component in the mixture noise, since the threshold values larger than $A+\max \left\{x_{i}\right\}$ result in error probabilities that are larger than 0.5 .
} 
First, a sufficient condition on the signal amplitude and the noise statistics is obtained for the sign detector not to be optimal.

Proposition 1: The sign detector is not an optimal singlethreshold detector, if the signal amplitude $A$ in (1) and the noise specified by (2) satisfy

$$
\sum_{i=1}^{M} \frac{w_{i}}{\sigma_{i}^{3}}\left(A+x_{i}\right) \mathrm{e}^{-\frac{\left(A+x_{i}\right)^{2}}{2 \sigma_{i}^{2}}}<0 .
$$

Proof: From (7), a first-order necessary condition for optimal $\tau$ value can be obtained by equating the first derivative with respect to $\tau$ to zero.

$$
\sum_{i=1}^{M} \frac{w_{i}}{\sqrt{2 \pi} \sigma_{i}}\left(-\mathrm{e}^{-\frac{\left(\tau-x_{i}+A\right)^{2}}{2 \sigma_{i}^{2}}}+\mathrm{e}^{-\frac{\left(-\tau+x_{i}+A\right)^{2}}{2 \sigma_{i}^{2}}}\right)=0 .
$$

Note that the condition in (9) is always satisfied by the sign detector, i.e., for $\tau=0$. In addition, the second derivative at $\tau=0$ can be calculated from (9) as

$$
\sum_{i=1}^{M} \frac{w_{i}}{\sqrt{2 \pi} \sigma_{i}^{3}}\left(\left(A-x_{i}\right) \mathrm{e}^{-\frac{\left(A-x_{i}\right)^{2}}{2 \sigma_{i}^{2}}}+\left(A+x_{i}\right) \mathrm{e}^{-\frac{\left(A+x_{i}\right)^{2}}{2 \sigma_{i}^{2}}}\right) .
$$

Due to the symmetry of the Gaussian mixture PDF, (10) is always negative when the condition in the proposition is satisfied. Since the first derivative is zero and the second derivative is negative at $\tau=0, \tau=0$ is a maximum point of the objective function in (7); hence, of the error probability in (6). Therefore, there exists $\tau \neq 0$ such that $\mathrm{P}(\tau)<\mathrm{P}(0)$, which proves that the sign detector is not optimal.

Proposition 1 provides a simple sufficient condition to determine if performance improvements can be obtained by using the optimal single-threshold detector instead of the sign detector. When the condition in (8) is satisfied, the optimal threshold $\tau_{\text {opt }}$ can be calculated from (7) (which is non-zero since the sign detector is not optimal), and the single-threshold detector with threshold $\tau_{\text {opt }}$ can be used for improved error performance. In addition, when the condition in Proposition 1 holds, the probability of error has a peak at $\tau=0$; hence, better (not necessarily optimal) thresholds than zero can be obtained simply by increasing $\tau$ from zero until the probability of error stops decreasing (cf. Fig. 3).

In addition to determining when the sign detector is not optimal, it is also important to specify when the sign detector is the optimal detector among all single-threshold detectors. In such a case, the optimization problem in (7) yields $\tau_{\text {opt }}=0$. In order to determine optimality conditions for the sign detector, it is first observed that as the variances of the Gaussian components in the mixture noise, specified by (2), go to infinity, $\mathrm{P}(\tau)$ in (6) converges to 0.5 for all finite $\tau$ values. Therefore, as $\sigma_{i}^{2} \rightarrow \infty$ for $i=1, \ldots, M, \frac{\mathrm{P}(0)}{\mathrm{P}\left(\tau_{\mathrm{opt}}\right)}$ becomes 1 ; that is, the sign detector and the optimal single-threshold detector converge to each other. Hence, for large variances, no significant difference between the error performances of the sign detector and the optimal single-threshold detector are expected.

Second, the following proposition presents a sufficient condition on the optimality of the sign detector based on the signal amplitude and the parameters of the Gaussian mixture noise.

Proposition 2: Assume that the signal amplitude $A$ in (1) is larger than or equal to the maximum of the mean values of the Gaussian components in the mixture noise specified by (2); that is, $A \geq \max _{i=1, \ldots, M}\left\{x_{i}\right\}$. Then, the sign detector is the optimal single-threshold detector.

Proof: Due to the symmetry of the Gaussian mixture noise, the first-order necessary optimality condition in (9) can be expressed as

$$
\begin{aligned}
& \sum_{i=1}^{M / 2} \frac{w_{i}}{\sigma_{i}}\left(\mathrm{e}^{-\frac{\left(-\tau+A+x_{i}\right)^{2}}{2 \sigma_{i}^{2}}}+\mathrm{e}^{-\frac{\left(-\tau+A-x_{i}\right)^{2}}{2 \sigma_{i}^{2}}}\right) \\
= & \sum_{i=1}^{M / 2} \frac{w_{i}}{\sigma_{i}}\left(\mathrm{e}^{-\frac{\left(\tau+A+x_{i}\right)^{2}}{2 \sigma_{i}^{2}}}+\mathrm{e}^{-\frac{\left(\tau+A-x_{i}\right)^{2}}{2 \sigma_{i}^{2}}}\right) .
\end{aligned}
$$

Since $A \geq \max _{i=1, \ldots, M}\left\{x_{i}\right\}, A+x_{i} \geq 0$ and $A-x_{i} \geq$ 0 for $i=1, \ldots, M$. Then, for $\tau<0$, it is observed that $\mathrm{e}^{-\frac{\left(-\tau+A+x_{i}\right)^{2}}{2 \sigma_{i}^{2}}}<\mathrm{e}^{-\frac{\left(\tau+A+x_{i}\right)^{2}}{2 \sigma_{i}^{2}}}$ and $\mathrm{e}^{-\frac{\left(-\tau+A-x_{i}\right)^{2}}{2 \sigma_{i}^{2}}}<$ $\mathrm{e}^{-\frac{\left(\tau+A-x_{i}\right)^{2}}{2 \sigma_{i}^{2}}}$ for $i=1, \ldots, M$. Therefore, the term on the right-hand-side (RHS) of (11) is always larger than that on the left-hand-side (LHS) for $\tau<0$. Similarly, it can be shown that the term on the LHS of (11) is always larger than that on the RHS for $\tau>0$. The equality is satisfied only when $\tau=0$. In addition, the second derivative at $\tau=0$, given in (10), is always positive since $A \pm x_{i} \geq 0$ for $i=1, \ldots, M$. Hence, $\tau=0$ is the unique minimum of (7).

Proposition 2 states that if the signal amplitude $A$ is larger than or equal to all the mean values of the Gaussian components in the mixture noise, then there is no need to search for the optimal threshold in (7) since $\tau_{\mathrm{opt}}=0$ in that case, which implies that the sign detector is the optimal singlethreshold detector. One practical application of Proposition 2 is related to binary detection in the presence of multipleaccess interference (MAI) [17]. In that case, (1) is given by $x=A_{1} b_{1}+\sum_{k=2}^{K} A_{k} b_{k}+\eta$ where $b_{i} \in\{ \pm 1\}$ and $\eta$ represents zero-mean Gaussian noise. The aim is to detect $b_{1}$ in the presence of MAI, $\sum_{k=2}^{K} A_{k} b_{k}$, and background noise, $\eta$, which together results in Gaussian mixture noise. Proposition 2 states that if $A_{1}>\sum_{k=2}^{K}\left|A_{k}\right|$, then the sign detector is the optimal single-threshold detector for this problem. As another application of Proposition 2, for impulsive noise, which is commonly modeled as a mixture of zero-mean Gaussian random variables with different variances, the sign detector is the optimal single-threshold detector since $\max _{i}\left\{x_{i}\right\}=0$ in that case.

If none of the conditions in Proposition 1 and Proposition 2 hold, then (7) can be solved to determine whether the sign detector is optimal. As an alternative approach, one can also determine the values of $\tau$ that satisfy (9), and, among those values, choose the one that minimizes the probability of error in (6) in order to determine $\tau_{\mathrm{opt}}$.

\section{Performance Analysis of Optimal SINGLE-THRESHOLD DETECTORS}

This section focuses on some properties of the optimal single-threshold detector and theoretical limits on its probability of error performance.

First, the average probability of error for the optimal singlethreshold detector is investigated as a function of the standard deviations of the Gaussian noise components in the Gaussian mixture noise specified by (2). Let $\boldsymbol{\sigma}=\left[\sigma_{1} \cdots \sigma_{M}\right]$ represent 
the standard deviation terms in (2). Then, the average probability of error for the optimal single-threshold detector can be expressed, from (6) and (7), as

$$
\begin{aligned}
& \mathrm{P}_{\mathrm{opt}}(\boldsymbol{\sigma})=\min _{\tau} \frac{1}{2} \sum_{i=1}^{M} w_{i} {\left[Q\left(\frac{\tau-x_{i}+A}{\sigma_{i}}\right)\right.} \\
&\left.+Q\left(\frac{-\tau+x_{i}+A}{\sigma_{i}}\right)\right] .
\end{aligned}
$$

For the sign detector, $\tau=0$ is used; hence, the average probability of error is given by

$$
\mathrm{P}_{\text {sign }}(\boldsymbol{\sigma})=\frac{1}{2} \sum_{i=1}^{M} w_{i}\left[Q\left(\frac{-x_{i}+A}{\sigma_{i}}\right)+Q\left(\frac{x_{i}+A}{\sigma_{i}}\right)\right] .
$$

For certain parameters of the Gaussian mixture noise, the average probabilities of error in (12) and (13) may not be monotonically decreasing as the standard deviations, $\sigma_{1}, \ldots, \sigma_{M}$, decrease. Although this might seem counter-intuitive at first, it mainly due to the multi-modal nature of the Gaussian mixture distribution. In Section V, numerical examples are provided to illustrate that behavior. Although the average probabilities of error can exhibit non-monotonic behaviors in general, the following proposition states that for equal standard deviations, a decrease in the standard deviation value can never result in an increase in the average probability of error for the optimal single-threshold detector. ${ }^{5}$

Proposition 3: Assume that $\sigma_{i}=\sigma$ for $i=1, \ldots, M$. Then, $\mathrm{P}_{\mathrm{opt}}(\boldsymbol{\sigma})$ in (12) is a monotone increasing function of $\sigma$.

Proof: When $\sigma_{i}=\sigma$ for $i=1, \ldots, M, \mathrm{P}_{\mathrm{opt}}(\boldsymbol{\sigma})$ in (12) is expressed as

$$
\begin{aligned}
\mathrm{P}_{\mathrm{opt}}(\sigma)=\frac{1}{2} \sum_{i=1}^{M} w_{i}\left[Q\left(\frac{\tau_{\mathrm{opt}}(\sigma)-x_{i}+A}{\sigma}\right)\right. \\
\left.+Q\left(\frac{-\tau_{\mathrm{opt}}(\sigma)+x_{i}+A}{\sigma}\right)\right] .
\end{aligned}
$$

where $\tau_{\text {opt }}(\sigma)$ represents the minimizer of (7), which satisfies the following first and second derivative conditions ${ }^{6}$

$$
\begin{aligned}
& \sum_{i=1}^{M} \frac{w_{i}}{\sigma}\left(\mathrm{e}^{-\frac{\left(\tau_{\text {opt }}(\sigma)-A-x_{i}\right)^{2}}{2 \sigma^{2}}}-\mathrm{e}^{\left.-\frac{\left(\tau_{\text {opt }}(\sigma)+A-x_{i}\right)^{2}}{2 \sigma^{2}}\right)}\right) 0, \\
& \sum_{i=1}^{M} \frac{w_{i}}{\sigma^{3}}\left[\left(-\tau_{\text {opt }}(\sigma)+A+x_{i}\right) \mathrm{e}^{-\frac{\left(-\tau_{\text {opt }}(\sigma)+A+x_{i}\right)^{2}}{2 \sigma^{2}}}\right. \\
&\left.+\left(\tau_{\text {opt }}(\sigma)+A-x_{i}\right) \mathrm{e}^{-\frac{\left(\tau_{\text {opt }}(\sigma)+A-x_{i}\right)^{2}}{2 \sigma^{2}}}\right]>0 .
\end{aligned}
$$

In order to prove the monotonicity of $\mathrm{P}_{\mathrm{opt}}(\sigma)$ in (14) with respect to $\sigma$, the first derivative of $\mathrm{P}_{\mathrm{opt}}(\sigma)$ is calculated as

\footnotetext{
${ }^{5}$ It can be shown that the result in Proposition 3 is valid also for asymmetric Gaussian mixture noise.

${ }^{6}$ Equations (15) and (16) can be obtained similarly to (9) and (10) by taking the derivatives of the summation term in (7) with respect to $\tau$.
}

follows:

$$
\begin{aligned}
& \frac{d \mathrm{P}_{\mathrm{opt}}(\sigma)}{d \sigma}=\frac{1}{2} \sum_{i=1}^{M} \frac{w_{i}}{\sqrt{2 \pi} \sigma^{2}} \\
& \times\left\{\left[\frac{d \tau_{\text {opt }}(\sigma)}{d \sigma} \sigma-\tau_{\text {opt }}(\sigma)+A+x_{i}\right] \mathrm{e}^{-\frac{\left(-\tau_{\mathrm{opt}}(\sigma)+A+x_{i}\right)^{2}}{2 \sigma^{2}}}\right. \\
& \left.-\left[\frac{d \tau_{\text {opt }}(\sigma)}{d \sigma} \sigma-\tau_{\text {opt }}(\sigma)-A+x_{i}\right] \mathrm{e}^{-\frac{\left(\tau_{\text {opt }}(\sigma)+A-x_{i}\right)^{2}}{2 \sigma^{2}}}\right\},
\end{aligned}
$$

which can be manipulated to obtain

$$
\begin{aligned}
& \frac{d \mathrm{P}_{\mathrm{opt}}(\sigma)}{d \sigma}=\frac{1}{2 \sqrt{2 \pi}} \frac{d \tau_{\mathrm{opt}}(\sigma)}{d \sigma} \sum_{i=1}^{M} \frac{w_{i}}{\sigma}\left[\mathrm{e}^{-\frac{\left(\tau_{\mathrm{opt}}(\sigma)-A-x_{i}\right)^{2}}{2 \sigma^{2}}}\right. \\
& \left.-\mathrm{e}^{-\frac{\left(\tau_{\text {opt }}(\sigma)+A-x_{i}\right)^{2}}{2 \sigma^{2}}}\right]+\frac{1}{2 \sqrt{2 \pi} \sigma^{2}} \sum_{i=1}^{M} w_{i}\left[\left(-\tau_{\text {opt }}(\sigma)+A+x_{i}\right)\right. \\
& \left.\mathrm{e}^{-\frac{\left(-\tau_{\mathrm{opt}}(\sigma)+A+x_{i}\right)^{2}}{2 \sigma^{2}}}+\left(\tau_{\mathrm{opt}}(\sigma)+A-x_{i}\right) \mathrm{e}^{-\frac{\left(\tau_{\mathrm{opt}}(\sigma)+A-x_{i}\right)^{2}}{2 \sigma^{2}}}\right] \text {. }
\end{aligned}
$$

Since $\tau_{\text {opt }}(\sigma)$ satisfies (15), the first term in (18) becomes zero. In addition, (16) implies that the second term in (18) is always positive. Therefore, $d \mathrm{P}_{\text {opt }}(\sigma) / d \sigma>0$ is satisfied; hence, $\mathrm{P}_{\mathrm{opt}}(\sigma)$ is a monotone increasing function of $\sigma$.

Proposition 3 states that for a single-threshold detector under symmetric Gaussian mixture noise with the same variance for all Gaussian components in the mixture (which is a well-suited model for communications systems with cochannel interference [3]), the probability of error decreases monotonically as the variance decreases if the detector uses the optimal threshold in (7). Therefore, as the signal-to-noise ratio (SNR) increases, the probability of error also decreases when the single threshold detector employs the optimal threshold. In other words, the optimization of the threshold for the singlethreshold detector provides such a desirable monotonicity property, which is not present in the sign detector in general (cf. Fig. 2).

Next, the behavior of the optimal single-threshold detector is investigated for very small variances. As $\sigma_{i} \rightarrow 0$ for $i=$ $1, \ldots, M$, the probability of error in (13) for the sign detector can be expressed as ${ }^{7}$

$$
\mathrm{P}_{\text {sign }}=\frac{1}{2} \sum_{i=1}^{M} w_{i} u\left(\left|x_{i}\right|-A\right),
$$

where $u(\cdot)$ is the unit step function defined as $u(x)=1$ for $x>0, u(x)=0.5$ for $x=0$, and $u(x)=0$ for $x<0$. Similarly, as $\sigma_{i} \rightarrow 0$ for $i=1, \ldots, M$, the average probability of error in (12) for the optimal single-threshold detector is given by

$$
\mathrm{P}_{\mathrm{opt}}=\min _{\tau} \frac{1}{2} \sum_{i=1}^{M} w_{i} u\left(\left|x_{i}-\tau\right|-A\right) .
$$

The expressions in (19) and (20) provide a simple interpretation of the average probability of error. For example, consider the values of $x_{1}, \ldots, x_{M}$ and $A$ as in Fig. 1-(a). Since the average probability of error expression in (19) states that the $x_{i}$ values that are outside the interval $(-A, A)$ contribute to the summation term, only the weights $w_{i+1}, \ldots, w_{M / 2}$

${ }^{7} x_{1}, \ldots, x_{M}$ are assumed to be distinct such that $\left|x_{j}-x_{k}\right| \gg \sigma_{i}$ as $\sigma_{i} \rightarrow 0, \forall j \neq k, \forall i$. 


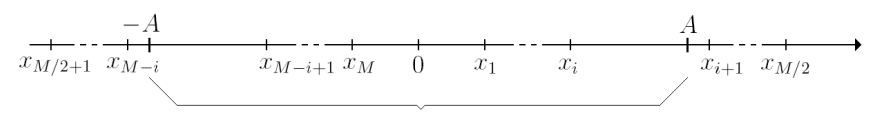

(a)

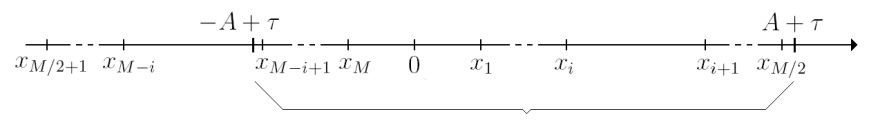

(b)

Fig. 1. (a) For the sign detector, the mean values ( $x_{j}$ 's) of the Gaussian mixture noise that are outside the interval $(-A, A)$ determine the average probability of error. (b) For the optimal single-threshold detector, the mean values $\left(x_{j}\right.$ 's) of the Gaussian mixture noise that are outside the interval $(-A+$ $\tau, A+\tau)$ determine the average probability of error.

and $w_{M / 2+1}, \ldots, w_{M-i}$ are employed in the calculation of the error probability for the settings in Fig. 1-(a). For the optimal single-threshold detector, various values of $\tau$ in (20) correspond to various shifts of the interval in Fig. 1-(a), as shown in Fig. 1-(b). Then, the value of $\tau$ that results in the minimum average probability of error is selected as the optimal threshold, $\tau_{\text {opt }}$.

The previous interpretation of the single-threshold detection for very small variances facilitates the calculation of a theoretical limit on performance improvements that can be achieved by using the optimal single-threshold detector instead of the sign detector.

Proposition 4: Let $0<x_{1}<\cdots<x_{M / 2}$ without loss of generality. As $\sigma_{i} \rightarrow 0$ for $i=1, \ldots, M$, the maximum ratio between the average probabilities of error for the sign detector and the optimal single-threshold detector, under symmetric Gaussian mixture noise given by (2), is specified as

$$
\max _{A, x_{1}, \ldots, x_{M}, w_{1}, \ldots, w_{M}} \frac{\mathrm{P}_{\text {sign }}}{\mathrm{P}_{\mathrm{opt}}}=2,
$$

which is achieved when there exists $i \in\{1, \ldots, M / 2-1\}$ such that $x_{i+1}>A>\left(x_{i}+x_{M / 2}\right) / 2{ }^{8}$

Proof: Let $x_{i}<A<x_{i+1}$ for any $i \in\{1, \ldots, M / 2-$ $1\} .{ }^{9}$ Then, the average probability of error for the sign detector can be calculated from (19) as $\mathrm{P}_{\text {sign }}=$ $\frac{1}{2}\left(\sum_{l=i+1}^{M / 2} w_{l}+\sum_{l=M / 2+1}^{M-i} w_{l}\right)$, which is equal to $\mathrm{P}_{\text {sign }}=$ $\sum_{l=i+1}^{M / 2} w_{l}$ due to symmetry property of the Gaussian mixture, i.e., $x_{i}=-x_{M-i+1}$ and $w_{i}=w_{M-i+1}$ for $i=1, \ldots, M / 2$. In order to obtain the maximum $\mathrm{P}_{\text {sign }} / \mathrm{P}_{\text {opt }}$ ratio, the parameter values that result in the minimum $\mathrm{P}_{\text {opt }}$ in (20) should be determined. The interpretation of the probability of error calculation related to the weights of $x_{j}$ 's that reside outside the interval $(-A+\tau, A+\tau)$ (cf. Fig. 1-(b)) implies that the maximum ratio can be obtained for a value of $\tau$ that results in a shift of the interval $(-A, A)$ in such a way that all the $x_{j}$ values that are on the shift direction are included in the new interval $(-A+\tau, A+\tau)$ in addition to the $x_{j}$ 's that are already in $(-A, A)$ (cf. Fig. 1). In that case, the average probability of error is given by $\mathrm{P}_{\mathrm{opt}}=\frac{1}{2} \sum_{l=M / 2}^{M-i}$, which is equal to $\mathrm{P}_{\mathrm{opt}}=\frac{1}{2} \sum_{l=i+1}^{M / 2}$ due to symmetry. Hence, it is obtained that $\mathrm{P}_{\text {opt }}=\mathrm{P}_{\text {sign }} / 2$, as claimed in the proposition.

\footnotetext{
${ }^{8}$ Note that the minimum value of $\mathrm{P}_{\text {sign }} / \mathrm{P}_{\text {opt }}$ is equal to one, which is achieved when the sign detector is the optimal single-threshold detector.

${ }^{9}$ There is no need to consider $i=M / 2$ since the sign detector is already optimal for $A>x_{M / 2}=\max \left\{x_{i}\right\}$, as stated in Proposition 2 .
}

Note that the scenario in Fig. 1-(b) can be obtained if $-A+$ $\tau<x_{M-i+1}$ and $A+\tau>x_{M / 2}$. Since $x_{M-i+1}=-x_{i}$, these inequalities imply $A>\left(x_{i}+x_{M / 2}\right) / 2$. As $A$ is assumed to satisfy $x_{i}<A<x_{i+1}$, the minimum probability of error can be obtained when $x_{i+1}>A>\frac{x_{i}+x_{M / 2}}{2}$, as stated in the proposition. ${ }^{10}$

By similar arguments, it can be shown that when $A=x_{i}$ for any $i \in\{1, \ldots, M / 2\}, \mathrm{P}_{\text {opt }}>\mathrm{P}_{\text {sign }} / 2$; hence, the maximum ratio cannot be obtained in the equality case. $\square$

The practical importance of Proposition 4 is that it sets an upper bound on the performance improvement that can be obtained by using the optimal single-threshold detector instead of the sign detector, when the variances of the Gaussian components in the mixture noise are significantly smaller than the distances between consecutive mean values, $x_{j}$ 's in (2). In such a case, Proposition 4 states that the optimal singlethreshold detector cannot have an average probability of error smaller than half of that for the sign detector. ${ }^{11}$

Proposition 4 also leads to the derivation of sufficient conditions for the sign detector to be optimal or not as $\sigma_{i} \rightarrow 0$ for $i=1, \ldots, M$. Two of them are stated below without any proofs.

- The sign detector is not optimal if there exists $i \in$ $\{1, \ldots, M / 2-1\}$ such that $A>\left(x_{i}+x_{i+1}\right) / 2$.

- For $x_{i}<A<x_{i+1}$, the sign detector in (4) is an optimal single-threshold detector if $A \leq\left(x_{i}+x_{i+1}\right) / 2$ and $w_{i} \geq$ $\sum_{l=i+1}^{M / 2} w_{l}$.

Remark 2: Although no fading is considered in the signal model in (1), the results in this study can be extended to slowly varying flat-fading channels under the assumption of perfect channel estimation, since the optimal threshold parameter can be calculated for each different channel realization in that case. On the other hand, if the duration between the updates of the threshold parameter is longer than the channel coherence time, then averages over fading statistics need to be taken in order to determine the optimal threshold. In that case, further analysis is required to extend the results of this study.

\section{NumERICAL RESUlts}

In this section, numerical examples are provided in order to investigate the theoretical results obtained in the previous sections. For all cases, the variances of the Gaussian components in the mixture noise are assumed to be the same; i.e., $\sigma_{i}=\sigma$ for $i=1, \ldots, M$ in (2).

First, symmetric Gaussian mixture noise with $M=10$ is considered, where the mean values of the Gaussian components in the mixture noise in (2) are specified as $\pm\left[\begin{array}{lllll}0.05 & 0.18 & 0.30 & 0.45 & 1.2\end{array}\right]$ with corresponding weights of [0.198 0.209 0.081 0.011 0.001]. Fig. 2 illustrates the average probabilities of error for the sign detector and the optimal single-threshold detector for various values of $A^{2} / \sigma^{2}$. The signal value $A$ in (1) is set to $A=1$, and $\sigma$ is varied in order to obtain various $A^{2} / \sigma^{2}$ values. From Fig. 2, significant performance improvement is observed for large $A^{2} / \sigma^{2}$ values

\footnotetext{
${ }^{10}$ For a leftwards shift, i.e., for $\tau<0,-A+\tau<x_{M / 2+1}=-x_{M / 2}$ and $A+\tau>x_{i}$ need to be satisfied for the maximum ratio, which results in the same expression.

${ }^{11}$ For asymmetric Gaussian mixture noise, the maximum ratio in Proposition 4 becomes infinity, since there can be cases in which the interval $(-A+\tau, A+\tau)$ in Fig. 1-(b) includes all the mean values ( $x_{j}$ 's) while the interval $(-A, A)$ in Fig. 1-(a) does not, which is possible due to the asymmetry of the mean values.
} 


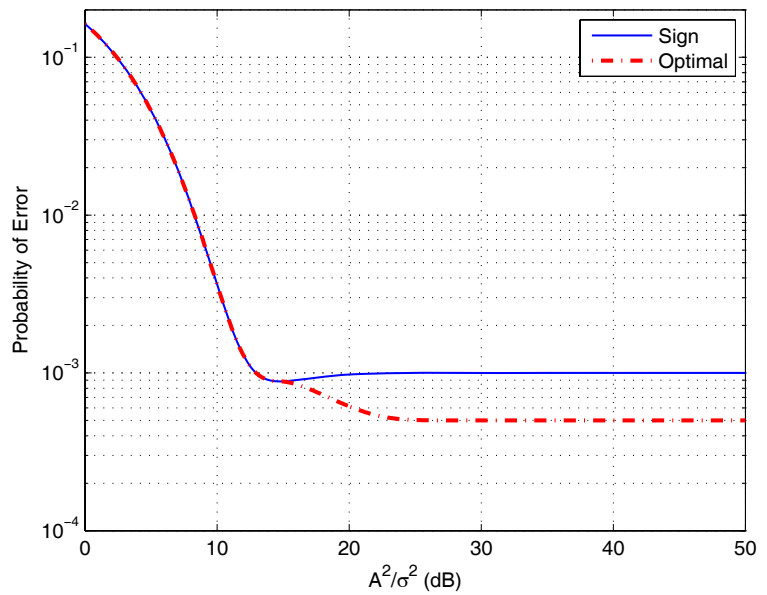

Fig. 2. Probability of error versus $A^{2} / \sigma^{2}$ for symmetric Gaussian mixture noise with $M=10$, where the center values are $\pm\left[\begin{array}{lllll}0.05 & 0.18 & 0.30 & 0.45 & 1.2\end{array}\right]$ with corresponding weights of $\left[\begin{array}{llllll}0.198 & 0.209 & 0.081 & 0.011 & 0.001\end{array}\right]$.

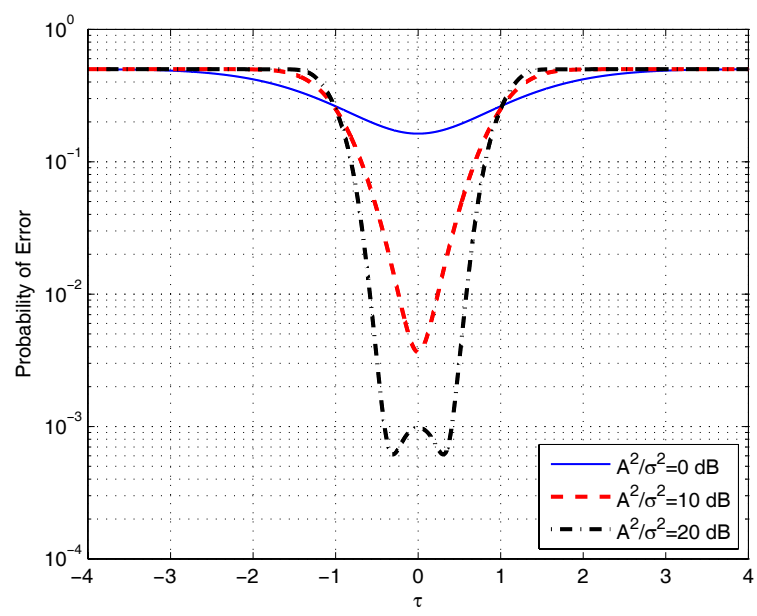

Fig. 3. Probability of error in (6) versus $\tau$ for various $A^{2} / \sigma^{2}$ values for the scenario in Fig. 2.

when the optimal single-threshold detector is used. On the other hand, for small $A^{2} / \sigma^{2}$ values; that is, for large $\sigma$ 's, the sign detector becomes optimal as expected (cf. Section III). In addition, the average probability of error for the optimal single-threshold detector reduces monotonically with $A^{2} / \sigma^{2}$, as predicted by Proposition 3. On the other hand, the sign detector exhibits a non-monotonic behavior and experiences a higher error floor. Finally, as $\sigma \rightarrow 0$, the ratio between the probabilities of error becomes $2\left(\mathrm{P}_{\text {sign }}=0.001\right.$ and $\left.\mathrm{P}_{\mathrm{opt}}=0.0005\right)$, which is expected from Proposition 4 , since $A$ satisfies the condition in the proposition, $x_{i+1}>A>$ $\left(x_{i}+x_{M / 2}\right) / 2$ for $i=4$ (namely, $1.2>1>(0.45+1.2) / 2=$ $0.825)$. In order to investigate the scenario in Fig. 2 in more detail, Fig. 3 plots the probability of error in (6) versus $\tau$ for various $A^{2} / \sigma^{2}$ values. It is again observed that as $\sigma^{2}$ increases, the sign detector becomes optimal.

Next, the effects of channel estimation errors on the performance of optimal single-threshold detection are investigated. For flat fading channels, the signal model in (1) can be

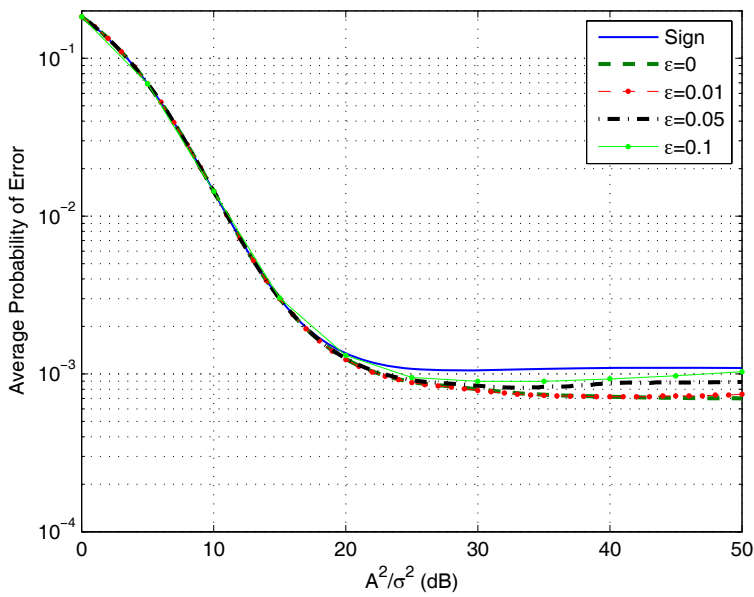

Fig. 4. Average probability of error versus $A^{2} / \sigma^{2}$ for the sign detector and the optimal single-threshold detector in the presence of channel estimation errors, where $\varepsilon$ denotes the standard deviation of the zero-mean Gaussian channel estimation error.

extended as $x=A \alpha b+n$, where $\alpha$ represents the channel coefficient. If the channel is known perfectly, then the effects of $\alpha$ can be removed via equalization and the model in (1) can still be used. However, the value of $\alpha$ is commonly obtained via channel estimation and its estimate can include certain errors. A common model for channel estimation errors is a zero-mean Gaussian random variable with standard deviation $\varepsilon$, and a generic probability distribution for the amplitude of the channel coefficient is the Nakagami- $m$ distribution [2]. In Fig. 4, the effects of channel estimation errors are investigated for an average power of unity for the Nakagami- $m$ channel coefficient, $m=4, A=1$, and the same Gaussian mixture noise parameters as in the previous scenario. It is observed that as the standard deviation of the channel estimation error, $\varepsilon$, increases, the performance difference between the sign detector and the optimal single-threshold detector decreases. This is expected since the threshold value that is obtained based on the channel estimate can become more different from the optimal threshold that is based on the true value of the channel coefficient.

Finally, symmetric Gaussian mixture noise with $M=$ 10 is considered with $\sigma_{i}=\sigma \forall i$, where the mean values of the Gaussian components are specified as $\pm\left[\begin{array}{lllll}2 & 4.8 & 5.4 & 7.5 & 9\end{array}\right]$ with corresponding weights of [0.35 0.099985 0.05 0.000005 0.00001]. In Fig. 5, the probabilities of error of the sign detector and the optimal singlethreshold detector are plotted versus SNR for $\sigma=0.1$, where SNR is defined as $\mathrm{SNR}=A^{2} /\left(\sigma^{2}+\sum_{i=1}^{M} w_{i} x_{i}^{2}\right)$. It is observed that the sign detector can be optimal or not optimal depending on the SNR value. For example, to achieve error probabilities of $10^{-5}$ and $9 \times 10^{-6}$, the sign detector needs respective increases of $0.55 \mathrm{~dB}$ and $1.5 \mathrm{~dB}$ in the SNR compared to the optimal single-threshold detector. A detailed investigation of the simulation results reveals that the sign detector is not optimal for $A \in(3.4,4.9), A \in(5.1,5.4)$ or $A \in(6.5,9)$. This is also in compliance with Proposition 2 , which states that the sign detector is optimal when the signal amplitude $A$ is larger than or equal to the maximum mean value of the Gaussian components in the mixture noise; 


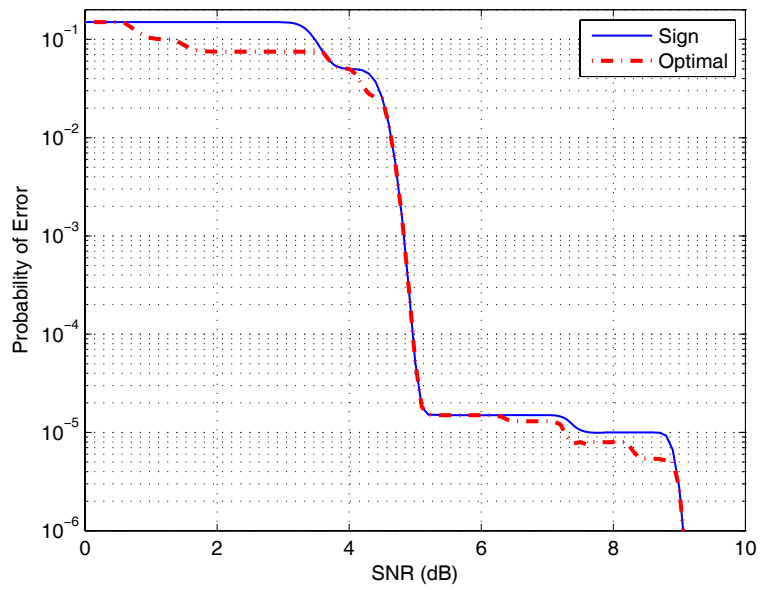

Fig. 5. Probability of error versus SNR for symmetric Gaussian mixture noise with $\sigma_{i}=\sigma=0.1$ for $i=1, \ldots, M$ and $M=10$.

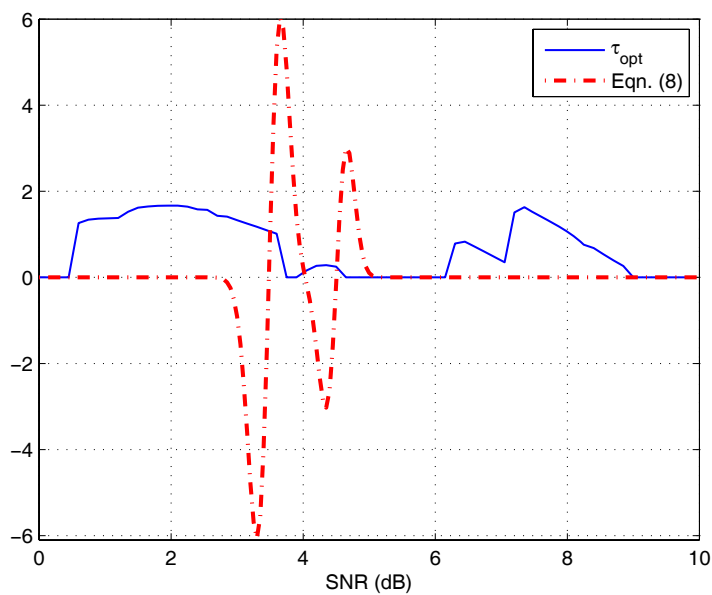

Fig. 6. The optimality function in (8) and the optimal threshold $\tau_{\text {opt }}$ in (7) versus SNR for the scenario in Fig. 5.

that is, $A \geq 9$ in this case. Fig. 6 illustrates the optimality function in (8) and $\tau_{\mathrm{opt}}$ in (7) versus SNR for this scenario. It is observed that whenever the optimality function in (8) is negative, the sign detector is not optimal (i.e., $\tau_{\text {opt }} \neq 0$ ) in accordance with Proposition 1. It is also noted that the condition in Proposition 1 is a sufficient but not a necessary condition for the sign detector not to be optimal, which can be observed, for example, at $\mathrm{SNR}=3.6 \mathrm{~dB}$, where the function value is positive and the sign detector is not optimal.

\section{CONCLUDING REMARKS}

In this paper, performance of single-threshold detectors has been investigated for binary communications systems under Gaussian mixture noise. Sufficient conditions have been obtained for the sign detector to be optimal or not optimal. Also, a monotonicity property of the error probability for the optimal single-threshold detector has been derived. In additional, a theoretical limit on the performance improvements that can be obtained by using the optimal single-threshold detector instead of the sign detector has been obtained. Finally, numerical examples have been provided to investigate the theoretical results.

\section{REFERENCES}

[1] H. V. Poor, An Introduction to Signal Detection and Estimation. New York: Springer-Verlag, 1994.

[2] J. G. Proakis, Digital Communications, 4th edition. New York: McGrawHill, 2001.

[3] V. Bhatia and B. Mulgrew, "Non-parametric likelihood based channel estimator for Gaussian mixture noise," Signal Process., vol. 87, pp. 2569-2586, Nov. 2007.

[4] C. Luschi and B. Mulgrew, "Nonparametric trellis equalization in the presence of non-Gaussian interference," IEEE Trans. Commun., vol. 51, pp. 229-239, Feb. 2003.

[5] A. Nasri, A. Nezampour, and R. Schober, "Adaptive $L_{p}$-norm diversity combining in non-Gaussian noise and interference," IEEE Trans. Wireless Commun., vol. 8, no. 8, pp. 4230-4240, Aug. 2009.

[6] C. Keller and M. Pursley, "Clipped diversity combining for channels with partial-band interference-part I: clipped-linear combining," IEEE Trans. Commun., vol. 35, pp. 1320-1328, Dec. 1987.

[7] P. Huber, Robust Statistics. New York: Wiley, 1981.

[8] T. Aysal and K. Barner, "Meridian filtering for robust signal processing," IEEE Trans. Signal Process., vol. 55, pp. 3949-3962, Aug. 2007.

[9] A. Nasri and R. Schober, "Adaptive $L_{p}$-norm metric for secondary BICM-OFDM systems," in Proc. IEEE Global Commun. Conf. (GLOBECOM 2009), HI, USA, Dec. 2009.

[10] G. Shevlyakov and K. Kim, "Robust minimax detection of a weak signal in noise with a bounded variance and density value at the center of symmetry," IEEE Trans. Inf. Theory, vol. 52, pp. 1206-1211, Mar. 2006.

[11] A. Swami, Q. Zhao, Y.-W. Hong, and L. Tong, editors, Wireless Sensor Networks: Signal Processing and Communications. Wiley, 2007.

[12] S. M. Kay, Fundamentals of Statistical Signal Processing: Detection Theory. Upper Saddle River, NJ: Prentice Hall, Inc., 1998.

[13] P. R. Prucnal and M. C. Teich, "Single-threshold detection of a random signal in noise with multiple independent observations - part 1: discrete case with application to optical communications," Applied Optics, vol. 17 , no. 22 , pp. $3576-3583$, Nov. 1978.

[14] T. Erseghe and S. Tomasin, "Optimized demodulation for MAI resilient UWB W-PAN receivers," in Proc. IEEE Int. Conf. Commun. (ICC), Beijing, China, May 2008, pp. 4867-4871.

[15] T. Erseghe, V. Cellini, and G. Dona, "On UWB impulse radio receivers derived by modeling MAI as a Gaussian mixture process," IEEE Trans. Wireless Commun., vol. 7, no. 6, pp. 2388-2396, June 2008.

[16] E. Ekrem, M. Koca, and H. Delic, "Robust ultra-wideband signal acquisition," IEEE Trans. Wireless Commun., vol. 7, no. 11, pp. 46564669, Nov. 2008.

[17] S. Verdu, Multiuser Detection, 1st edition. Cambridge, UK: Cambridge University Press, 1998.

[18] S. M. Kay, J. H. Michels, H. Chen, and P. K. Varshney, "Reducing probability of decision error using stochastic resonance," IEEE Signal Process. Lett., vol. 13, no. 11, pp. 695-698, Nov. 2006. 